\title{
AGC-based SU Selection for Cooperative Spectrum Sensing with Direct-Conversion Cognitive Radio
}

\author{
Dayan Adionel Guimarães
}

\begin{abstract}
A direct-conversion receiver for cooperative spectrum sensing (CSS) was recently proposed to assess the performances of eigenvalue-based detectors and the energy detector (ED). In this paper, the problem of secondary user (SU) selection for CSS is solved by a classification method based on the automatic gain control loop gains of the receivers. Low complexity detectors are compared using this method: the Gerschgorin radii and centers ratio, the Gini index detector, the Pietra-Ricci index detector, and the locally most powerful invariant test. The ED is also included as a benchmark. Significant performance improvements are achieved in comparison with the use of the same number of SUs without selection.
\end{abstract}

Keywords-Cooperative spectrum sensing, direct-conversion receiver, dynamic spectrum access, secondary user selection.

\section{INTRODUCTION}

Nowadays, the high demand for new telecommunications services has been a major technology development driver, which can be noticed, for instance, by the recent advances of the Internet of things (IoT) and the fifth generation (5G) of communication networks. Discussions on the sixth generation (6G) have also started in this context. However, due to the current fixed bandwidth allocation policy, which grants spectrum usage right to a licensed or primary user (PU), the radio-frequency (RF) spectrum scarcity has become a major bottleneck. The dynamic spectrum access (DSA) is foreseen as a solution for spectrum sharing between PUs and unlicensed cognitive radio secondary users (SUs), exploiting the fact that the RF spectrum is considerably underutilized, given that much of the time and in certain regions there are allocated frequency bands that are unoccupied [1]. Thus, SU transmissions can be carried out simultaneously with PU transmissions, as long as no harmful interference is caused to the primary network, or in a non-overlapping manner, taking advantage of transmission opportunities in licensed bands that are momentarily unoccupied.

One of the enabling technologies of DSA is spectrum sensing [2], which confers to an SU the capability of identifying vacant bands for DSA. In spectrum sensing, the decision upon the presence (hypothesis $\mathcal{H}_{1}$ ) or absence (hypothesis $\mathcal{H}_{0}$ ) of the PU signal in the monitored band is made by comparing a test statistic with a decision threshold set according with the target sensing performance. Well-known test statistics are

Dayan Adionel Guimarães is with the National Institute of Telecommunications (Inatel), PO Box 05, 37540-000 Santa Rita do Sapucaí - MG - Brazil (Tel:+55 (35) 3471 9227, Fax:+55 (35) 3471 9314, e-mail: dayan@inatel.br)

This work was partially supported by RNP, with resources from MCTIC, Grant No. No 01245.010604/2020-14, under the 6G Mobile Communications Systems project of the Radiocommunication Reference Center (Centro de Referência em Radiocomunicações, CRR) of Inatel. based on energy detection, matched filtering, cyclostationary feature detection, and eigenvalue-based detection [2].

If each SU makes spectrum sensing independently of the others, unreliable decisions may result, mostly due to channel fading, shadowing and unreachable (hidden) SUs. In cooperative spectrum sensing (CSS), in which a group of SUs collaborate, the spatial diversity achieved by SUs in different locations is explored, yielding more reliable decisions [3].

Among the cooperation strategies, the focus of this paper is the data-fusion centralized CSS. In this strategy, the received signal samples collected by the SUs are forwarded to a fusion center (FC), where the test statistic is formed and the global decision on the occupation state of the sensed band is made. When a global decision is made in favor of a vacant band, it is broadcast to the SUs to allow the subsequent DSA by means of an ordinary or specific access protocol.

The spectrum sensing performance is measured by the probability of false alarm, $P_{\mathrm{fa}}=\operatorname{Pr}\left\{T>\gamma \mid \mathcal{H}_{0}\right\}$, and the probability of detection, $P_{\mathrm{d}}=\operatorname{Pr}\left\{T>\gamma \mid \mathcal{H}_{1}\right\}$, where $\operatorname{Pr}\{\cdot\}$ denotes the probability of occurrence of the underlying event, $T$ is the test statistic formed according with the adopted detection technique, and $\gamma$ is the decision threshold. It is desirable a low $P_{\mathrm{fa}}$ to increase the chance of opportunistic spectrum access by the SUs, which increases the secondary network throughput. A high $P_{\mathrm{d}}$ is also desired to protect the primary network from interference caused by the secondary network when it mistakenly detects a vacant band and uses it. Since the objectives of increasing $P_{\mathrm{d}}$ and reducing $P_{\mathrm{fa}}$ are concurrent, a trade-off solution ruled by a standard is typically adopted in practice. For example, the IEEE 802.22 standard establishes $P_{\mathrm{fa}} \leq 0.1$ and $P_{\mathrm{d}} \geq 0.9$ [4].

\section{A. Related research and contributions}

A direct-conversion receiver (DCR) model for centralized data-fusion CSS has been proposed in [5], called implementation-oriented model. The development of such model was motivated by the fact that there were (and still there is) a lack of research results that show the influence of typical receiver circuitry on the spectrum sensing performance. The DCR architecture has been chosen for being the most adequate choice for software-defined radio receivers and monolithic integration [6], [7], thus carrying a state-of-theart appeal. However, a DCR is prone to impairments such as I\&Q imbalance, flicker noise and direct-current-offset (DCoffset) [7], [8].

It has been concluded in [5] that when spectrum sensing does not take into account such circuitry-related impairments, 
its performance can be overestimated when compared with the implementation-oriented model. The main process that degrades performance was found in [5] to be the automatic gain control (AGC). An improved version of such model was proposed in [9], where a fine-tuning of the original model is made and several other new performance results were presented, taking into consideration other detectors besides the ED and eigenvalue-based ones.

Recently, low-complexity detectors for spectrum sensing were proposed. The computation of their test statistics are slightly more complex than the energy detector (ED), since the test statistics are formed directly from the elements of the received signal sample covariance matrix (SCM). The detectors are the locally most powerful invariant test (LMPIT) [10], the Gerschgorin radii and centers ratio (GRCR) detector [11], the Gini index detector (GID) [12], and the Pietra-Ricci index detector (PRIDe) [13]. These detectors are also robust against received signal and noise power variations over time, attain the constant false alarm rate (CFAR) property, can outperform many state-of-the-art detectors, and are blind in the sense that neither any PU signal characteristics nor the noise variance information are needed to form the test statistics.

The choice of the detection technique is not the unique problem in cooperative spectrum sensing. Another fundamental problem is the selection of the SUs to cooperate, aiming at optimizing performance with a minimum of users to save control channel resources (bandwidth and energy), and to reduce communication overhead and reporting delay. Several strategies can be found in the literature to solve the SU selection problem. The strategy proposed in [14] is based on the evaluation of the signal correlation experienced by the cognitive radio users. The method proposed in [15] also explores correlations to perform SU selection. In [16], the SU selection algorithm is based on the SU velocity stability and on the correlation of users' signals. Algorithms for estimating the number of sources are applied in [17] to solve the SU selection problem. In [18], SU selection aims at maximizing the network throughput, an optimization problem that is solved based on SUs' energy harvesting, sensing, and reporting attributes.

In this paper, the problem of SU selection for CSS is solved by means of a classification method based on the AGC loop gains of DCR receivers. The performances of the detectors LMPIT, GRCR, GID, PRIDe and ED are assessed under this method, demonstrating that it can bring significant improvements with low implementation complexity.

The remainder of the paper is organized as follows: Section II describes the DCR receiver model. The proposed AGCbased SU selection method is presented in Section III. The detectors whose performance are compared under the proposed SU selection method are listed in Section IV. Section V is devoted to the numerical results and discussions. The conclusions are drawn in Section VI.

\section{DCR-BASED MODEL}

As the name suggests, a DCR receiver directly converts the passband received signal into baseband. Figure 1 shows a typical DCR architecture [7]. The signal received by the antenna goes through a low noise amplifier (LNA) and then through a wideband band-pass filter (WBPF) responsible for limiting the input signal to the bandwidth of interest. A power splitter drives the in-phase and quadrature branches where the direct-conversion to baseband is made by the pair of mixers and local carriers generated by the frequency synthesizer. DCoffset compensation blocks try to eliminate the static part of the DC-offset, often keeping a residual dynamic part. Lowpass filters (LPFs) limit the sensed channel bandwidth and avoids aliasing. The variable-gain amplifiers (VGAs), which are part of the AGC loop, keep the signal within the dynamic range of the analog-to-digital converters (ADCs). The digital signal processor (DSP) does the control tasks, computes the test statistic and performs the decision on the occupation state of the sensed band.

Taking into consideration the main processes or impairments that degrade the spectrum sensing performance in a DCR receiver, the $\mathrm{FC}$ receiver model derived from Figure 1 is given in Figure 2. Further details fully discussed in [9] are omitted here for conciseness. In this model, $\mathbf{y}_{i}^{\mathrm{T}}$ denotes the $i$-th row, $i=1, \ldots, m$, of the signal matrix $\mathbf{Y} \in \mathbb{C}^{m \times n}$ received at the $\mathrm{FC}$, which is

$$
\mathbf{Y}=\mathbf{H X}+\mathbf{V}
$$

where $\mathbf{H} \in \mathbb{C}^{m \times s}$ is the channel matrix defining the gains between $s$ PU transmitters and $m$ SU receivers. Matrix $\mathbf{X} \in$ $\mathbb{C}^{s \times n}$ contains the $n$ samples of the $s$ PUs, and matrix $\mathbf{V} \in$ $\mathbb{C}^{m \times n}$ contains the zero-mean Gaussian noise samples whose variance is determined according to the average signal-to-noise ratio (SNR). Under $\mathcal{H}_{0}, \mathbf{Y}=\mathbf{V}$. Under $\mathcal{H}_{1}, \mathbf{Y}=\mathbf{H X}+\mathbf{V}$.

Matrix $\mathbf{H}$ is defined in [9] to model a Ricean fading channel with random Rice factor, as typically found in real environments. Specifically, the Rice factor is Gaussian-distributed with mean $\mu_{K}$ and standard deviation $\sigma_{K}$, both in $\mathrm{dB}$ [19]. Time-varying average received signal powers across the SUs are modeled by making the signal levels vary with uniform distribution around the average, by a fraction $\rho_{\mathrm{S}}$ of this average. Matrix V models time-varying average noise powers across the SUs, also uniformly-distributed around the average, by a fraction $\rho_{\mathrm{N}}$ of this average.

To model filtering effects, each row of $\mathbf{Y}$ becomes the result of low-pass moving-average (MA) filtering of the PU signal and noise samples. The filter has configurable discrete impulse response length, $L$, which controls the time correlation in the samples. The residual dynamic DC-offset is taken into account by the summation following the MA filters. The variance of the DC-offset samples, $\sigma_{\mathrm{dc}}^{2}$, is determined according to the signal-to-DC-offset ratio (SDCR). In $\mathrm{dB}$, $\mathrm{SDCR}=10 \log _{10}\left(p_{\mathrm{avg}} / \sigma_{\mathrm{dc}}^{2}\right)$.

The combined effect of any amplification and gain control present in the signal path is modeled by the AGC block in Figure 2. At the $i$-th $\mathrm{SU}, i=1,2, \ldots, m$, this gain is

$$
g_{i}=\frac{f_{\mathrm{od}} \sqrt{2 n}}{6\left\|\mathbf{y}_{i}\right\|},
$$

where $\|\cdot\|$ denotes the Euclidean norm, and the overdrive factor $f_{\text {od }}$ models different levels of signal clipping caused by the ADCs [5]. These gains have the role of guaranteeing that 


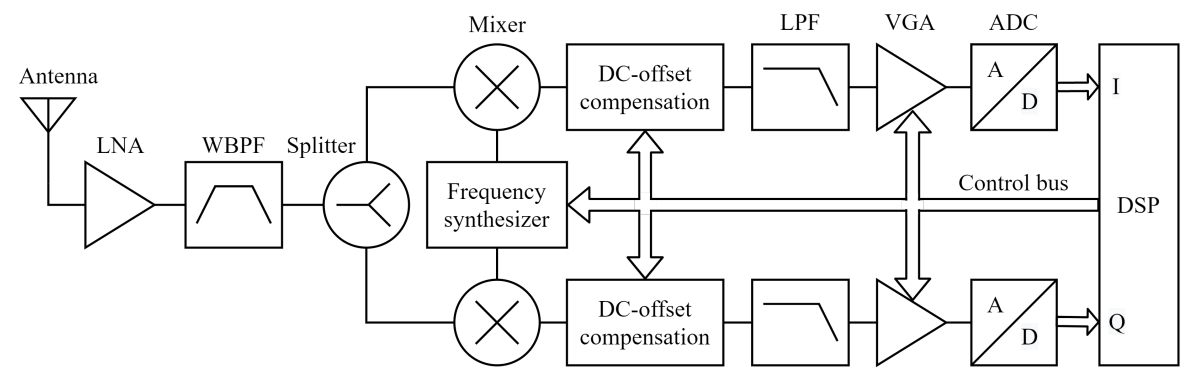

Fig. 1. Direct-conversion receiver architecture. Adapted from [7].

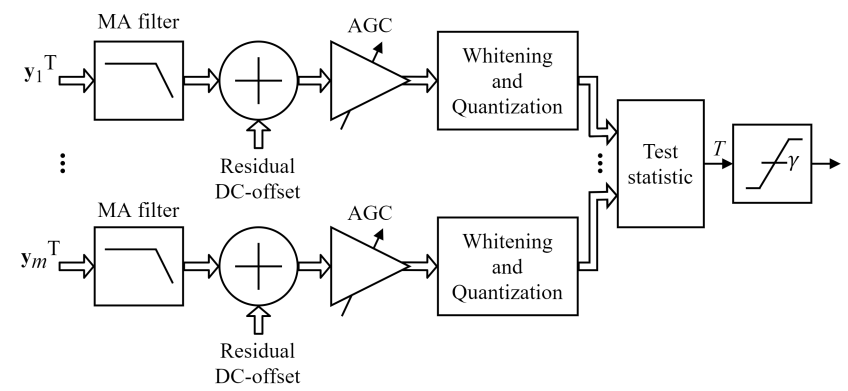

Fig. 2. Fusion center receiver model presented in [9].

almost the total signal excursion fits the dynamic range of the ADCs, going below or above this range as determined by $f_{\text {od }}$.

Whitening decorrelates the AGC output samples to improve spectrum sensing performance. The whitened samples are then quantized and possibly clipped to model the ADCs' effect. It is subsumed that whitening makes use of high resolution quantization (using floating-point arithmetic), while the samples resulted from whitening are quantized with a resolution as small as possible, the smallest sufficient for not causing significant spectrum sensing performance degradation [9].

After applying the above processing to all rows of $\mathbf{Y}$, the test statistic $T$ of the detector adopted in the FC is formed and compared with the decision threshold $\gamma$, yielding the decision upon the occupation of the monitored band.

\section{AGC-BASED SU SELECTION}

Out of $M$ candidate SUs, the $m$ selected SUs are those for which $g_{i}$, as defined in (2) with $m$ replaced by $M$, are the $m$ smallest. It means that, $m$ out of the $M$ rows of $\mathbf{Y}$ are used, and are the rows whose indexes correspond to the indexes of the $m$ smallest $g_{i}$ from the set $\left\{g_{1}, g_{2}, \ldots, g_{M}\right\}$. This method is consistent with normal reasoning, since a small $g_{i}$ corresponds to a high received signal level, i.e. a high value of $\left\|\mathbf{y}_{i}^{\mathrm{T}}\right\|$ in (2).

In terms of realization of the method, if the $M$ candidate SUs transmit their collected sample values to the FC and the SU selection is made at the FC, the method can be seen as a censoring scheme. In this case the potentially high number of transmissions to the FC will demand higher control complexity and coordination tasks, and more resources of control channels. On the other hand, the $M$ SUs that are in conditions to cooperate, for instance those registered in the secondary base station of interest, can share their AGC gain metrics and decide which subset of SUs will send their sample values to the FC. In this selective report scheme, the SUFC control channel overhead diminishes, at the cost of the need of control channel links among the SUs. Another option is the transmission of the AGC gains to the FC before the transmission of the collected samples. The FC then selects the best subset of SUs based on the received AGC gains, and the corresponding SUs are requested to report their sample values. This alternative seems to require less control channel resources, but creates the need of splitting the total report interval into a pre-report and the report itself.

\section{ANALYZED DETECTORS}

The test statistics of the detectors whose performances are analyzed in Section V are listed in what follows. The ED test statistic under the DCR-based model is

$$
T_{\mathrm{ED}}=\sum_{i=1}^{m} \frac{1}{g_{i}^{2} \sigma_{i}^{2}} \sum_{j=1}^{n}\left|y_{i j}\right|^{2}
$$

where $y_{i j}$ is the element on the $i$-th row and $j$-th column of the received signal matrix $\mathbf{Y}$ defined in (1), and $\sigma_{i}^{2}$ is the noise variance at the input of the $i$-th SU. The gain $g_{i}$ defined in (2) is needed to correct the noise variances altered by the AGC.

In the case of the LMPIT detector [10], the test statistic is

$$
T_{\text {LMPIT }}=\sum_{i=1}^{m} \sum_{j=1}^{m}\left|c_{i j}\right|^{2},
$$

where $c_{i j}$ is the element on the $i$-th row and $j$-th column of the matrix $\mathbf{C}=\mathbf{E}^{-1 / 2} \mathbf{R} \mathbf{E}^{-1 / 2}$, for $i, j=1,2, \ldots, m$, where $\mathbf{E}$ is the diagonal matrix with diagonal elements $d_{i i}=r_{i i}$, where $r_{i i}$ are the elements on the main diagonal of the SCM

$$
\mathbf{R}=\frac{1}{n} \mathbf{Y} \mathbf{Y}^{\dagger}
$$

where $\dagger$ denotes complex conjugate and transpose.

The computation of $T_{\text {LMPIT }}$ is quite simple, since $\mathbf{C}$ results from a matrix multiplication, with $\mathbf{E}^{-1 / 2}$ being quite simple as well, given that $\mathbf{E}$ is diagonal. Thus, most of the computational complexity of the LMPIT refers to the computation of $\mathbf{R}$.

The test statistic of the GRCR detector [11] is

$$
T_{\mathrm{GRCR}}=\left(\sum_{i=1}^{m} r_{i i}\right)^{-1} \sum_{i=1}^{m} \sum_{j=1, j \neq i}^{m}\left|r_{i j}\right|,
$$

where $r_{i j}$ is the element on the $i$-th row and $j$-th column of the matrix $\mathbf{R}$. 
The test statistics of the GID [12] and the PRIDe [13] detectors are respectively computed according to

$$
\begin{gathered}
T_{\mathrm{GID}}=\left(\sum_{i=1}^{m^{2}} \sum_{j=1}^{m^{2}}\left|r_{i}-r_{j}\right|\right)^{-1} \sum_{i=1}^{m^{2}}\left|r_{i}\right|, \\
T_{\mathrm{PRIDe}}=\left(\sum_{i=1}^{m^{2}}\left|r_{i}-\bar{r}\right|\right)^{-1} \sum_{i=1}^{m^{2}}\left|r_{i}\right|,
\end{gathered}
$$

where $r_{i}$, for $i=1,2, \ldots, m^{2}$, is the $i$-th element of the vector $\mathbf{r}$ formed by stacking the columns of $\mathbf{R}$, and $\bar{r}=\left(1 / m^{2}\right) \sum_{i=1}^{m^{2}} r_{i}$.

The computations of $T_{\mathrm{GRCR}}, T_{\mathrm{GID}}$ and $T_{\mathrm{PRIDe}}$ are similar to each other and simple, even simpler than the computation of $T_{\text {LMPIT }}$. In the case of the former three, the most complex operation is associated with the computation of $\mathbf{R}$.

\section{NUMERICAL RESULTS}

This section presents receiver operating characteristic (ROC) curves, which trades $P_{\mathrm{d}}$ versus $P_{\mathrm{fa}}$ as the decision threshold is varied. Each point on all curves has been determined from 20000 Monte Carlo computer simulation runs, corresponding to the generation of the same number of each test statistic under $\mathcal{H}_{0}$ and $\mathcal{H}_{1}$. The Matlab simulation code used to generate the results is available in [20].

The system parameters, which were chosen to carry practical significance [9], are: number of PUs, $s=1$; number of selected SUs, $m=6$; number of candidate SUs, $M=$ 12 (when the selection method is in operation) and $M=$ 6 (selection method off); number of samples, $n=200$; average signal-to-noise ratio across the SUs, SNR $=-10$ $\mathrm{dB}$; signal-to-DC-offset ratio, $\mathrm{SDCR}=5 \mathrm{~dB} ; 3$ quantization bits; overdrive factor, $f_{\text {od }}=1.2$; filter impulse response length, $L=20$; uniformly-distributed received signal power variation about the average, with variation fraction $\rho_{\mathrm{S}}=0.9$; uniformly-distributed noise power variation about the average, with variation fraction $\rho_{\mathrm{N}}=0.2$; mean and standard deviation of the channel Rice factor, $\mu_{K}=1.88 \mathrm{~dB}$ and $\sigma_{K}=4.13$ $\mathrm{dB}$, respectively, representing a urban environment [19]. The extreme case of pure Rayleigh fading $\left(\mu_{K}=-\infty \mathrm{dB}\right.$ and $\sigma_{K}=0 \mathrm{~dB}$ ) has been analyzed as well.

Figure 3 shows results for the Ricean sensing channel, for the SU selection method in operation $(M=12, m=6$, solid lines), and the selection method off ( $M=6, m=6$, dashed lines). It can be seen that selection has improved the performance of all detectors. The improvement achieved by the ED was enough to put it the top position, whereas this position was occupied by the PRIDe in the case of no SU selection. The worst performance was achieved by the GID in both cases.

Figure 4 shows results for the Rayleigh sensing channel, for the SU selection method in operation $(M=12, m=6$, solid lines), and the selection method off ( $M=6, m=6$, dashed lines). The improvements due to SU selection is also evident. The ranking was maintained from the case of no SU

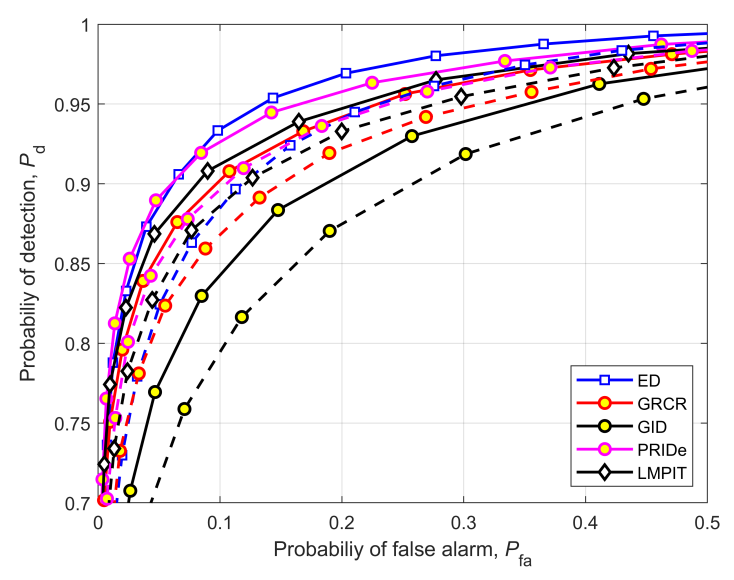

Fig. 3. Performance results for the Ricean channel. Selection method in operation ( $M=12, m=6$, solid lines); selection method off $(M=6$, $m=6$, dashed lines). Better viewed in color.

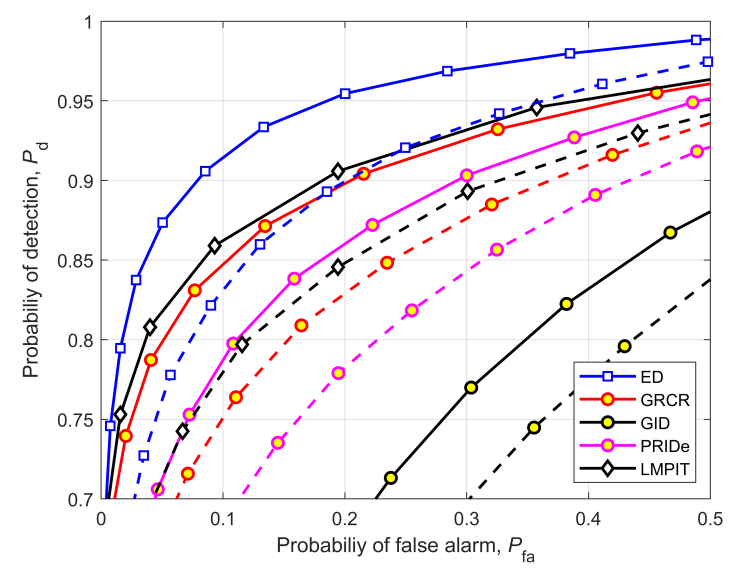

Fig. 4. Performance results for the Rayleigh channel. Selection method in operation $(M=12, m=6$, solid lines); selection method off ( $M=6$, $m=6$, dashed lines). Better viewed in color.

selection to the case of SU selection in operation, with the ED occupying the first position and the last occupied by the GID.

The solid lines in Figure 5 correspond to the same parameters considered in Figure 3, but now the dashed lines refer to the conventional system model, in which there is no circuitryrelated signal processing operations on the elements of $\mathbf{Y}$. The performances of the ED, the GRCR, the PRIDe and the LMPIT are clustered close to each other, with a slight advantage of the conventional model. The situation is reverted in the case of the GID, whose performance stayed considerably far below the others. Comparing the positions of the dashed ROC curves produced under the conventional model in Figure 3 with those achieved by the DCR-based model in Figure 5, the previouslymentioned overestimation of the performance is readily seen in the latter case. This overestimation is more pronounced for the ED, as pointed out in [9].

The conclusions drawn from Figure 5 with respect to the overestimation of the performance when the conventional model is adopted can be also extracted from Figure 6, where the Rayleigh fading channel is considered. However, now this overestimation was not enough for outperforming the DCRbased model with SU selection. 


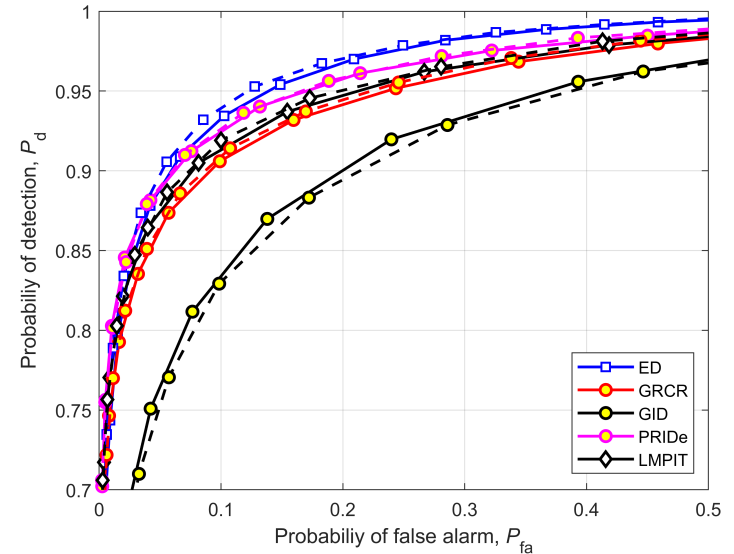

Fig. 5. Performance results for the Ricean channel. Selection method in operation $(M=12, m=6$, solid lines); selection method off $(M=6$, $m=6$, conventional model, dashed lines). Better viewed in color.

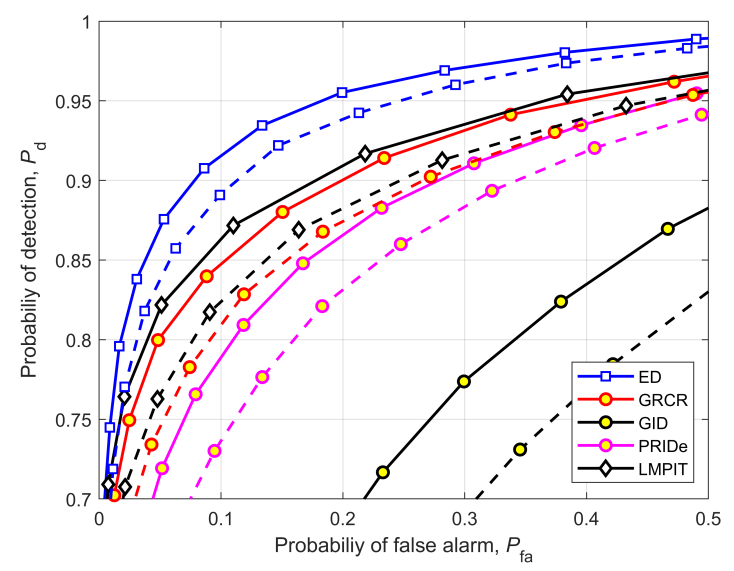

Fig. 6. Performance results for the Rayleigh channel. Selection method in operation ( $M=12, m=6$, solid lines); selection method off ( $M=6$, $m=6$, conventional model, dashed lines). Better viewed in color

\section{CONCLUSIONS}

The problem of secondary user selection for data-fusion cooperative spectrum sensing was solved in this paper by applying a classification method based on the automatic gain control loop gains of direct-conversion receivers. Low complexity detectors were compared using this method: the Gerschgorin radii and centers ratio, the Gini index detector, the Pietra-Ricci index detector, and the locally most powerful invariant test. The energy detector has been also included as a benchmark. Significant performance improvements were reported in comparison with the use of the same number of SUs without any selection method.

A natural extension of the present research consists of assessing the performance of the AGC-based SU selection method in different scenarios and system parameters, perhaps considering other state-of-the-art detectors independent of their implementation complexities.

\section{REFERENCES}

[1] M. A. McHenry, P. A. Tenhula, D. McCloskey, D. A. Roberson, and C. S. Hood, "Chicago spectrum occupancy measurements \& analysis and a long-term studies proposal," in Proc. of the first Int. workshop on technology and policy for accessing spectrum. ACM, 2006, doi: $10.1145 / 1234388.1234389$.

[2] Y. Arjoune and N. Kaabouch, "A comprehensive survey on spectrum sensing in cognitive radio networks: Recent advances, new challenges, and future research directions," Sensors, vol. 19, no. 1, 2019 , doi: 10.3390/s19010126. [Online]. Available: http://www.mdpi.com/ $1424-8220 / 19 / 1 / 126$

[3] I. F. Akyildiz, B. F. Lo, and R. Balakrishnan, "Cooperative spectrum sensing in cognitive radio networks: A survey," Elsevier Physical Comm., vol. 4, pp. 40-62, mar 2011, doi: 10.1016/j.phycom.2010.12.003

[4] The Institute of Electrical and Electronic Engineers, IEEE. (2011) IEEE 802 Part 22: Cognitive Wireless RAN Medium Access Control (MAC) and Physical Layer (PHY) Specifications: Policies and Procedures for Operation in the TV Bands. [Online]. Available: http://standards.ieee.org/getieee802/download/802.22-2011.pdf

[5] D. A. Guimarães and R. A. A. de Souza, "Implementation-oriented model for centralized data-fusion cooperative spectrum sensing," IEEE Commun. Lett., vol. 16, no. 11, pp. 1804-1807, 2012, doi: 10.1109/LCOMM.2012.092112.121614.

[6] J. Laskar and S. Chakraborty, Modern receiver front-ends: Systems, Circuits, and Integration. Hoboken, NJ, USA: Wiley-Interscience, 2004. [Online]. Available: http://www.loc.gov/catdir/bios/wiley046/ 2003019188.html

[7] S. Spiridon, Toward 5G Software Defined Radio Receiver Front-Ends, ser. SpringerBriefs in Electrical and Computer Engineering. Springer International Publishing, 2016, doi: 10.1007/978-3-319-32759-4.

[8] B. Razavi, "Design considerations for direct-conversion receivers," IEEE Transactions on Circuits and Systems-Part II: Analog and Digital Signal Processing, vol. 44, no. 6, pp. 428-435, Jun. 1997, doi: $10.1109 / 82.592569$.

[9] D. A. Guimarães and E. J. T. Pereira, "Performance of detectors for spectrum sensing using a direct-conversion receiver model," J. Commun. and Inf. Systems, May 2021, Submitted.

[10] D. Ramirez, J. Via, I. Santamaria, and L. L. Scharf, "Locally most powerful invariant tests for correlation and sphericity of gaussian vectors," IEEE Transactions on Information Theory, vol. 59, no. 4, pp. 2128 2141, April 2013, doi: 10.1109/TIT.2012.2232705.

[11] D. A. Guimarães, "Robust test statistic for cooperative spectrum sensing based on the Gerschgorin circle theorem," IEEE Access, vol. 6, pp. 2445-2456, 2018, doi: 10.1109/ACCESS.2017.2783443.

[12] — , "Gini index inspired robust detector for spectrum sensing over Ricean channels," Electron. Lett., November 2018, doi: 10.1049/el.2018.7375.

[13] _ - "Pietra-Ricci index detector for centralized data fusion cooperative spectrum sensing," IEEE Trans. Veh. Technol., vol. 69, no. 10, pp. 12354-12 358, 2020, doi: 10.1109/TVT.2020.3009440.

[14] A. S. Cacciapuoti, I. F. Akyildiz, and L. Paura, "Correlation-aware user selection for cooperative spectrum sensing in cognitive radio ad hoc networks," IEEE Journal on Selected Areas in Communications, vol. 30, no. 2, pp. 297-306, 2012.

[15] Y. Zhang, W. Zhao, D. Wang, D. Zhai, and X. Tang, "Correlation based secondary users selection for cooperative spectrum sensing network," in IoT as a Service, B. Li, C. Li, M. Yang, Z. Yan, and J. Zheng, Eds. Cham: Springer International Publishing, 2021, pp. 68-79.

[16] M. Duan, Z. Zeng, C. Guo, and F. Liu, "User selection for cooperative spectrum sensing in mobile cognitive radios," in 2015 IEEE/CIC International Conference on Communications in China (ICCC), 2015, pp. $1-6$.

[17] A. Stefanovska, K. Gao, and B. Shen, "EEF criterion based user selection for cooperative spectrum sensing in cognitive radio network," in 2016 Eighth International Conference on Ubiquitous and Future Networks (ICUFN), 2016, pp. 16-21.

[18] A. Celik, A. Alsharoa, and A. E. Kamal, "Hybrid energy harvestingbased cooperative spectrum sensing and access in heterogeneous cognitive radio networks," IEEE Transactions on Cognitive Communications and Networking, vol. 3, no. 1, pp. 37-48, 2017.

[19] S. Zhu, T. S. Ghazaany, S. M. R. Jones, R. A. Abd-Alhameed, J. M. Noras, T. Van Buren, J. Wilson, T. Suggett, and S. Marker, "Probability distribution of Rician $K$-factor in urban, suburban and rural areas using real-world captured data," IEEE Trans. Antennas Propag., vol. 62, no. 7, pp. 3835-3839, Jul 2014, doi: 10.1109/TAP.2014.2318072.

[20] D. A. Guimarães, "Matlab code for AGC-based SU selection for cooperative spectrum sensing with direct-conversion cognitive radio," June 2021, XXXIX Simpósio Brasileiro de Telecomunicações e Processamento de Sinais, 2021. [Online]. Available: https://www. dropbox.com/s/afkl3nyms21pvin/AGC based_SU selection.m?dl=0 